\title{
Trastuzumab is not a tyrosine kinase inhibitor
}

Nishant Mohan and Wen Jin Wu

We read with great interest the Review by Ewer, M. S. \& Ewer, S. M. (Cardiotoxicity of anticancer treatments. Nat. Rev. Cardiol. 12 , 547-558; 2015), ${ }^{1}$ because it is highly relevant to our research. We appreciate the thorough review of the heart disorders and the associated molecular mechanisms elicited by receptor tyrosine-protein kinase erbB-2 (ERBB2)-targeted agents in this article. However, we believe that the authors' description of trastuzumab as "the first monoclonal antibody tyrosine kinase inhibitor" is conceptually incorrect and does not reflect the current state of literature in this field.

Tyrosine kinase inhibitors (TKIs), such as lapatinib, inhibit the kinase activity of epidermal growth factor receptor (EGFR), and ERBB2 and ligand-induced receptor phosphorylation by reversibly binding to the ATP-binding site of the kinase. ${ }^{2}$ Cetuximab, a therapeutic monoclonal antibody directed against the extracellular domain of EGFR, acts as a TKI to block the activation of EGFR tyrosine kinase by competing for ligand binding. ${ }^{3,4}$ Trastuzumab is a therapeutic monoclonal antibody directed against the extracellular domain of ERBB2. ${ }^{5}$ However, the effects of trastuzumab on ERBB2 kinase activity are different from those of TKIs and cetuximab. An anti-ERBB2 monoclonal antibody 4D5, which was later humanized to generate trastuzumab, was shown to exhibit agonistic signalling, resulting in increased ERBB2 phosphorylation that correlated with its inhibitory effect on ERBB2overexpressing breast cancer cells. ${ }^{6}$ Growing evidence indicates that targeting ERBB2 using trastuzumab increases ERBB2 tyrosine phosphorylation in trastuzumab-sensitive breast cancer cells. ${ }^{7-9}$ Diemeier and colleagues reported that the inhibitory effect of trastuzumab on trastuzumab-sensitive breast cancer cells is associated with its capacity to induce ERBB2 phosphorylation at Tyr1248. ${ }^{7}$ Gijsen and co-workers also reported that trastuzumab induced ERBB2 tyrosine phosphorylation, ${ }^{8}$ and together with another group observed that trastuzumab treatment induced paradoxical activation of mitogenactivated protein kinase $1 / 3$ in breast cancer cells. ${ }^{89}$ In 2014, we demonstrated that trastuzumab upregulated ERBB2 tyrosine kinase activity in trastuzumab-sensitive breast cancer cells, and that this upregulated activity contributed to ERBB2 phosphorylation. ${ }^{9}$

Despite different effects on ERBB2 phosphorylation, both lapatinib and trastuzumab have the capacity to downregulate Akt activity and to inhibit growth of breast cancer cells. Based on our data, we postulate that binding of trastuzumab to ERBB2 could induce structural changes in the ERBB2 cytoplasmic kinase domain, leading to allosteric activation of ERBB2 kinase and transphosphorylation of ERBB2.9,10 Trastuzumab-induced ERBB2 phosphorylation provides docking sites for downstream signalling molecules, which are different from those induced by a ligand (such as epidermal growth factor), in that they recruit signalling molecules such as Csk-homologous kinase, a non-receptor tyrosine kinase. Negative signals downstream of ERBB2 subsequently contribute to growth inhibition of ERBB2-positive breast cancer cells. ${ }^{9} 10$ Taken together, these studies provide evidence that trastuzumab does not function as a TKI.

Division of Biotechnology Research and Review I, Office of Biotechnology Products, Office of Pharmaceutical Quality, Center for Drug Evaluation and Research, U.S. Food and Drug Administration, 10903 New Hampshire Avenue, Silver Spring, MD 20993, USA (N.M., W.J.W.).
Correspondence to: W.J.W. wen.wu@fda.hhs.gov

\section{Acknowledgements}

We thank J. Zhou and T. S. Stantchev from the Center for Drug Evaluation and Research, U.S. Food and Drug Administration, for critical review of the manuscript.

\section{Competing interests}

N.M. and W.J.W are employees of the US Food and Drug Administration. This Correspondence reflects the views of the authors and should not be construed to represent FDA's views or policies.

1. Ewer, M. S. \& Ewer, S. M. Cardiotoxicity of anticancer treatments. Nat. Rev. Cardiol. 12, 547-558 (2015).

2. Medina, P. J. \& Goodin, S. Lapatinib: a dual inhibitor of human epidermal growth factor receptor tyrosine kinases. Clin. Ther. 8, 1426-1447 (2008).

3. Mendelsohn, J. \& Baselga, J. Epidermal growth factor receptor targeting in cancer. Semin. Oncol. 33, 369-385 (2006).

4. Mendelsohn, J. \& Baselga, J. The EGF receptor family as targets for cancer therapy. Oncogene 19, 6550-6565 (2000).

5. Hudis, C. A. Trastuzumab-mechanism of action and use in clinical practice. N. Engl. J. Med. 357, 39-51 (2007).

6. Scott, G. K. et al. p185HER2 signal transduction in breast cancer cells. J. Biol. Chem. 266, 14300-14305 (1991).

7. Diermeier, S. et al. Epidermal growth factor receptor coexpression modulates susceptibility to Herceptin in HER2/neu overexpressing breast cancer cells via specific erbB-receptor interaction and activation. Exp. Cell. Res. 304, 604-619 (2005).

8. Gijsen, M. et al. HER2 phosphorylation is maintained by a PKB negative feedback loop in response to anti-HER2 herceptin in breast cancer. PLoS Biol. 8, e1000563 (2010).

9. Dokmanovic, M. et al. Trastuzumab-induced recruitment of Csk-homologous kinase (CHK) to ErbB2 receptor is associated with ErbB2-Y1248 phosphorylation and ErbB2 degradation to mediate cell growth inhibition. Cancer Biol. Ther. 8, 1029-1041 (2014).

10. Dokmanovic, M. \& Wu, W. J. Trastuzumabinduced HER2 phosphorylation: exploring the mechanisms and implications. Receptors Clin. Investig. 1, e340 (2014). 